\title{
The Distribution of Zinc in Low-Friction ZDDP Tribofilm on Iron Oxide
}

\author{
${\text { Kosuke } \text { Ito }^{1,2)^{*}} \text {, Koshi Adachi }}^{1)}$, Minoru Goto ${ }^{3)}$, and Koji Kato ${ }^{1,4)}$ \\ ${ }^{1)}$ Tribology Laboratory, Graduate School of Engineering, Tohoku University \\ 6-6-01 Aoba, Aramaki, Aoba-ku, Sendai, Miyagi 980-8579, Japan \\ ${ }^{2)}$ Current affiliation: Laboratory of Tribology and System Dynamics, Ecole Centrale de Lyon \\ 36 avenue Guy de Collongue, 69134 Ecully Cedex, France \\ ${ }^{3)}$ Department of Mechanical Enginnering, Ube National College of Technology \\ 2-14-1 Tokiwadai, Ube, Yamaguchi 755-8555, Japan. \\ ${ }^{4)}$ Current affiliation: Department of Mechanical Engineering, College of Engineering, Nihon University \\ 1 Naka-gawara, Tokusada, Tamura-machi, Koriyama, Fukushima 963-8642, Japan \\ * Corresponding author: kito@tribo.mech.tohoku.ac.jp
}

( Manuscript received 30 June 2008; accepted 10 December 2008; published 15 January 2009 )

\begin{abstract}
To develop a low-friction and low-wear tribo-system for engine components, the authors have been studying the ZDDP tribofilm formed on iron oxide. Tribotests on iron-oxide / steel sliding pairs were performed in PAO with 1 mass\% ZDDP. It was confirmed that the friction coefficient was lower in the PAO with ZDDP than that in pure PAO. The distributions of elements on the wear track of the iron oxide were analyzed by EPMA and found that a zinc-containing layer was distributed on almost entire contact surface.
\end{abstract}

Keywords: friction, water-vapor treatment, iron oxide, zinc dialkyldithiophosphate, ZDDP, tribofilm

\section{Introduction}

The reduction of $\mathrm{CO}_{2}$ emissions by improving the fuel economy is a strong social requirement for internal combustion engines. Sliding engine components with low frictional loss lead to such an improvement. On the other hand, the reduction of $\mathrm{NO}_{\mathrm{x}}$ emissions is another strong requirement. Some of the $\mathrm{NO}_{\mathrm{x}}$ reduction technologies, however, are known to increase the wear of engine components ${ }^{1)}$. Therefore, it is important to develop a tribo-system that can meet the both requirements.

It has been proven by engine tests that the application of an iron oxide layer on the sliding surface by water-vapor treatment is effective to reduce the wear ${ }^{1,2}$. In addition to the wear reduction, it has been reported in the previous study ${ }^{3)}$ that the application of such an iron oxide layer can lead to a low friction tribo-system in the lubricant that contains an anti-wear additive, Zinc dialkyldithiophosphate (ZDDP).

The structure and composition of the tribofilm on the iron oxide was analyzed by TEM with EDX and found that they were different from those on the steel ${ }^{3}$. The most significant difference was that the tribofilm on the iron oxide had a multi-layered structure with "zinc-containing layer(s)," while that on steel did not.
More specifically, the tribofilm can be divided into three layers: the topmost, middle, and bottom layers. The top and bottom layer included zinc ( $\mathrm{Zn})$, iron (Fe), sulfur (S), phosphorous $(\mathrm{P})$, and oxygen $(\mathrm{O})$ (i.e. zinc-containing layer), which is unique to the tribofilm on the iron oxide. By TEM cross-sectional observation on several locations on the wear track, the thickness of the top and bottom layer have been found to be few $\mathrm{nm}$ and 10 to $30 \mathrm{~nm}$, respectively. The middle layer included mostly $\mathrm{P}$ and $\mathrm{O}$ with almost no $\mathrm{Zn}$ and $\mathrm{S}$ and thought to be a phosphate layer, which is similar to the tribofilm on steel. The $\mathrm{P}$ concentration in the middle and bottom layers was similar. The thickness of the middle layer was two to few times thicker than that of the bottom layer. In some cases actually observed, the middle layer was missing, but the zinc-containing layer was found in all the locations that had observed. The total thickness of the tribofilm ranged from 30 to $130 \mathrm{~nm}$. More detailed description can be found elsewhere ${ }^{3)}$.

Such a unique tribofilm should have been originated from the nature of the iron oxide and is believed to be the key to the low friction. It was not clear however, if the results of TEM/EDX analyses above described the general characteristics of the ZDDP tribofilm on the iron oxide because the areas analyzed were so localized, as is often the case with such a nano-scopic method. For such 


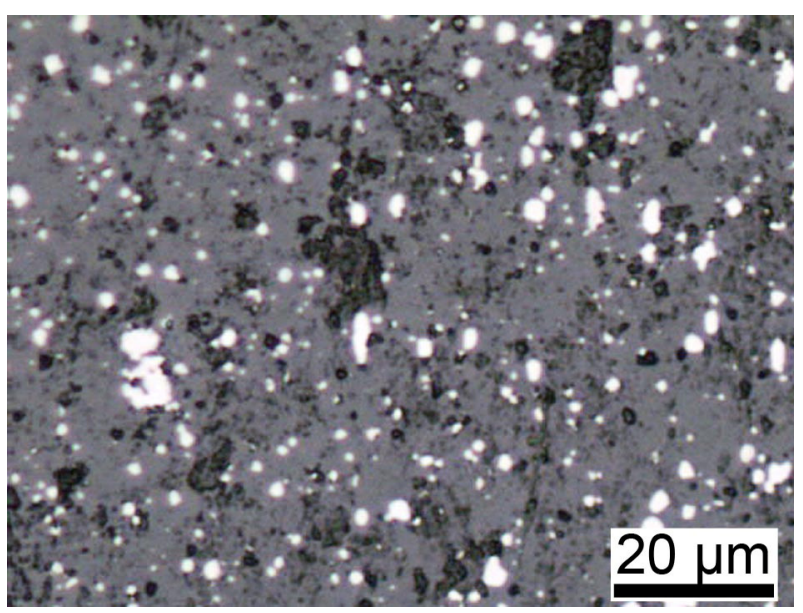

Fig. 1 An optical microscope photograph of an iron oxide plate before test

a reason, the relation between the mechanism of low friction and the unique structure of the tribofilm has not been discussed in detail. Therefore, it is important to identify the general characteristics of the tribofilm that may be related to the low friction.

The objective of this study, therefore, is to confirm if the unique tribofilm is distributed widely on the wear track on the iron oxide. Tribotests are performed under the same condition as the previous study ${ }^{3)}$ and the wear track is analyzed by EPMA to identify the distribution of the elements. The results are compared with the findings of the previous study ${ }^{3)}$ and how it can be related with the low friction is discussed.

\section{Test procedure}

A cylinder-on-flat, oscillating type test apparatus, Cameron Plint tribo-tester, was used for the friction experiments. The cylinder specimen slid on the plate specimen in oscillating motion. The oscillating frequency of the cylinder specimen was $7 \mathrm{~Hz}$ with a stroke of $7 \mathrm{~mm}$. The total test duration was $1 \mathrm{hr}$. The load was $50 \mathrm{~N}$ during the initial 5 minutes, and then increased to $350 \mathrm{~N}$ for the rest of the period.

The cylinder type specimen is $6 \mathrm{~mm}$ in diameter and $6 \mathrm{~mm}$ in length. It is made of NF 100C6 bearing steel that is equivalent of JIS SUJ2 or ASTM 52100. The Vickers hardness of the cylinder is $8.1 \mathrm{GPa}$. The sliding surfaces of the cylinders were polished using emery papers of $\# 800$, \#1200, \#2000, and \#4000, and then finished with diamond slurry with $1-\mu \mathrm{m}$ grains. The resultant surface roughness was $0.05 \mu \mathrm{m} \mathrm{Ra}$.

The plate specimen has dimension of $10 \times 8 \times 2 \mathrm{~mm}$. It is made of high-speed tool steel, JIS SKH51 that is equivalent of ASTM M2. The initial sliding surfaces of the plates were miller finished. The initial Rockwell hardness (in $\mathrm{C}$ scale) of the plate was 64 . Then a layer of iron oxide was formed on the surfaces by the water-vapor treatment at $600{ }^{\circ} \mathrm{C}$ for 2 hours. Details on the process can be found elsewhere ${ }^{1,2)}$. After the treatment, the

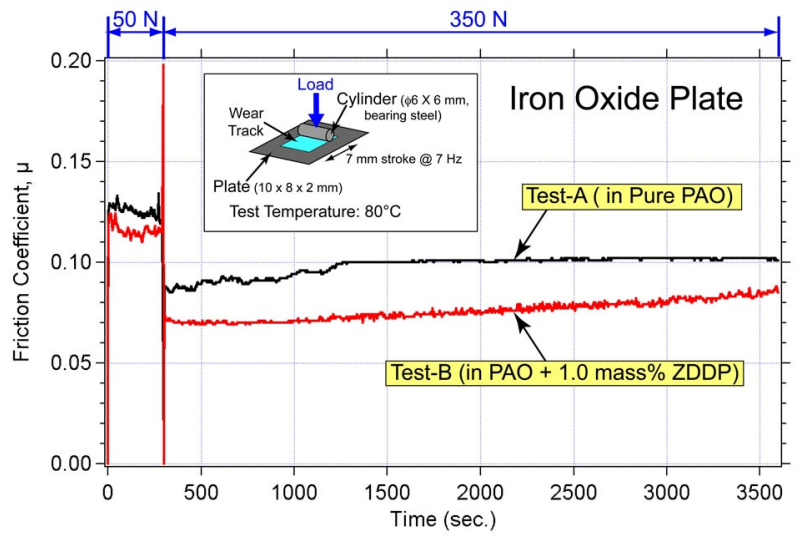

Fig. 2 Friction coefficient of iron-oxide / steel pairs in PAO with or without 1 mass\% ZDDP

surface was polished with diamond slurry with $1-\mu \mathrm{m}$ grains. The resultant surface roughness was $0.1 \mu \mathrm{m}$ Ra. The oxide layer after the polishing was 2 to $5 \mu \mathrm{m}$ in thickness and contained about 50 mass $\%$ of $\mathrm{Fe}_{3} \mathrm{O}_{4}$ and 50 mass $\%$ of $\mathrm{FeO}$. An optical microscope photograph of the plate is shown in Fig. 1. The white spots on the surface are hard phase particles that are dispersed in the steel to sustain the high temperature hardness.

The plate specimen was soaked in the oil bath that was filled with a lubricant. The temperature of the lubricant was kept at $80^{\circ} \mathrm{C}$. Two types of lubricants were used: pure poly-alpha-olefine (PAO 6) and PAO 6 mixed with 1.0 mass $\%$ of ZDDP (4-methyl-2-pentanol) for Tests-A and -B, respectively as summarized in Table 1 . PAO is one of the most typical synthetic base oils for engine oil.

The maximum Hertzian contact pressure is $850 \mathrm{MPa}$ and the minimum oil film thickness calculated in the middle of the stroke by the simplified Dowson-Higginson equation $^{4-6)}$ is $0.02 \mu \mathrm{m}$. Because the calculated oil film thickness is smaller than the surface roughness, the condition is in the mixed lubrication regime.

After the experiments, the specimens were cleaned in heptane for 10 minutes by using an ultrasonic cleaner. Then the wear tracks of the plates and cylinders were observed under an optical microscope. Electron probe microanalysis (EPMA) was conducted to identify the distributions of chemical elements on the plate surface.

Table 1 Test Lubricants

\begin{tabular}{lcc}
\hline & Test-A & Test-B \\
\hline Base Oil & PAO 6 & PAO 6 \\
Additive & None & 1.0 mass $\%$ ZDDP \\
\hline
\end{tabular}

\section{Test results}

The friction coefficient data of the Tests-A and $-\mathrm{B}$ is plotted in Fig. 2. (The result of Test-A is the one reported in the previous study ${ }^{3)}$, which is presen ted 
(a)

(b)
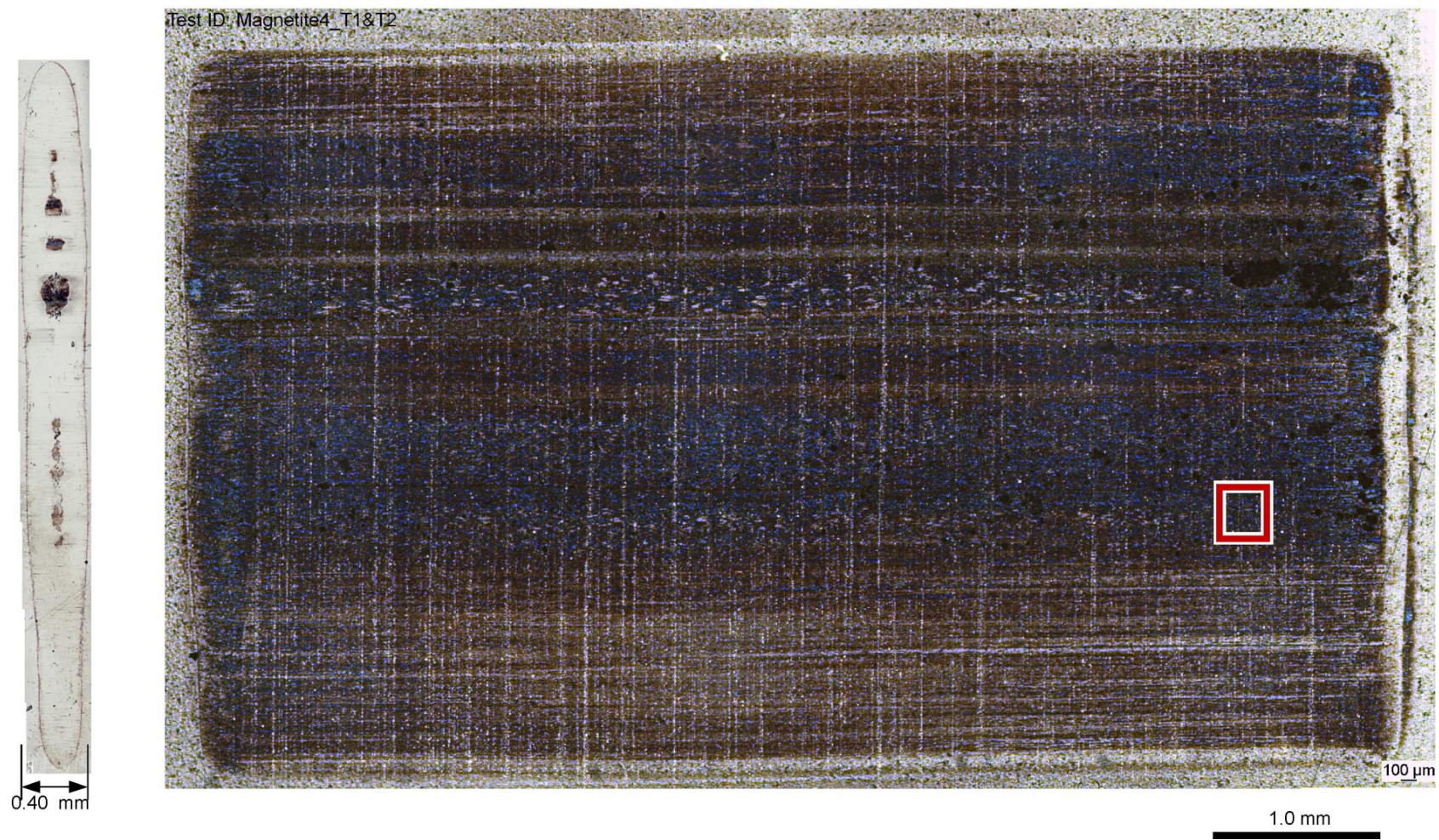

Fig. 3 Optical microscope photographs of the (a) steel cylinder and (b) iron oxide plate, respectively, after Test-B. The red square corresponds to the area analyzed by EPMA (Fig. 5)

again as a reference.) In general, the result of Test-B is a typical one under the same condition and consistent with the previous study ${ }^{3)}$. The friction coefficient, $\mu$, reaches to a steady value of 0.10 after $1300 \mathrm{sec}$. in pure PAO (i.e. Test-A). With ZDDP (i.e. Test-B), $\mu$ is always lower than that in pure $\mathrm{PAO}$, which reaches to the

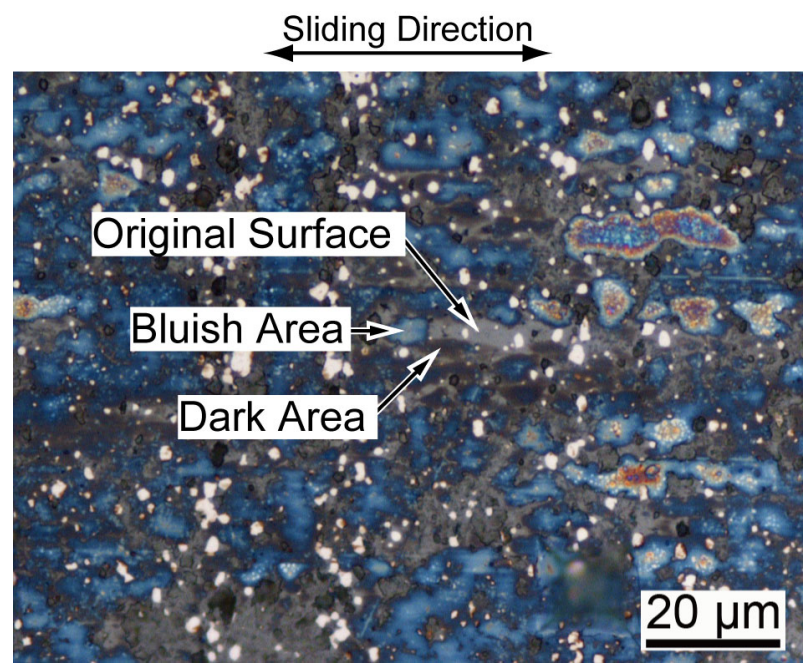

Fig. 4 An optical microscope photograph of the iron oxide plate after Test-B lowest value of 0.07 at around $500 \mathrm{sec}$. and reaches to 0.09 at the end of the 1-hr test. The maximum wear depth of the plate of Test-A was less than $0.6 \mu \mathrm{m}$, which is of the same magnitude as the peak-to-valley roughness of the initial surface thus not large enough to define the wear volume. Not even a measurable change due to wear was observed by profilometry on the plate of Test-B.

The optical microscope photographs of the cylinder and plate of Test-B are shown in Fig. 3. The wear track of the plate is severely discolored. An enlarged view of the wear track of the plate is shown in Fig. 4. Bluish and dark areas can be seen. Some areas appear as orange.

The chemical element mapping results by EPMA on the plate of Test-B are shown in Fig. 5 together with the optical microscope photograph of the analyzed area as Fig. 5(a). The area shown in Fig. 5(a) corresponds to the red square in Fig. 3. The red rectangle in Fig. 5(a) corresponds to the view area of Fig. 4. The distribution of oxygen was almost feature-less (not shown). No Zn, S, nor $\mathrm{P}$ was detected in the gray areas (i.e. uncontacted areas). The distributions of $\mathrm{Zn}, \mathrm{S}$, and $\mathrm{P}$ correspond to the discolored areas (i.e. orange, dark, or bluish areas) in the optical image. No specific color corresponds to the intensities of any specific element. It appears that the color rather represents the intensities of the elements, 


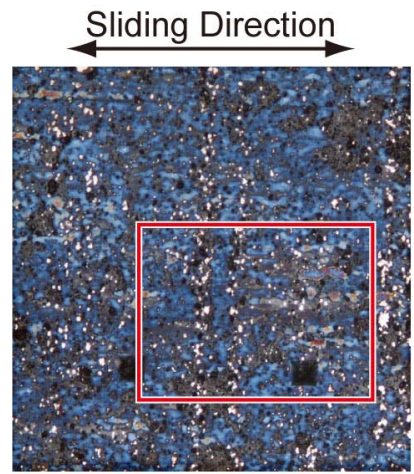

(a) Optical Image

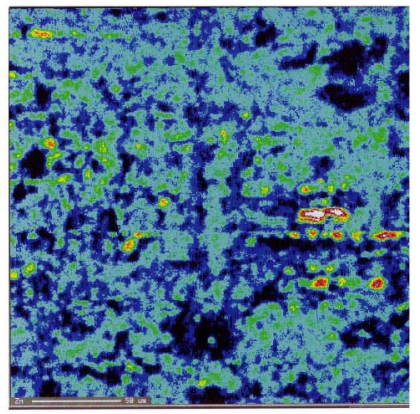

(b) Zn

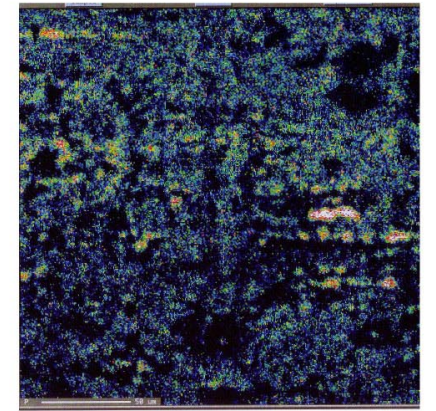

(c) $\mathrm{P}$

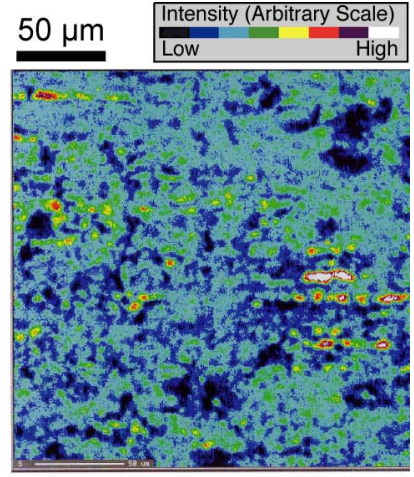

(d) $S$

Fig. 5(a) An optical microscope photographs of the iron oxide plate after Test-B and distributions of (b) zinc, (c) phosphorous, and (d) sulfur, respectively, analyzed by EPMA on the view area of (a). The red rectangle in (a) corresponds to the view area of Fig. 4

suggesting the difference in the thickness rather than the chemical composition. Less strong intensities of each element are observed from the dark areas, suggesting the tribofilm of such areas is thinner than that of the orange and bluish ones in this particular case. Because $\mathrm{Zn}, \mathrm{S}$, and $\mathrm{P}$ are included in ZDDP and there is no other source of the elements, the discolored areas are the tribofilm originated from ZDDP. In fact, the discolored area was not seen outside the wear track.

\section{Discussions}

The results of this study have confirmed that $\mathrm{Zn}$ and $\mathrm{S}$ always exist in the discolored area (i.e. the tribofilm). This is consistent with the result of the TEM/EDX analyses on limited points in the previous study ${ }^{3)}$. It is now reasonable to state that the tribofilm on the iron oxide always includes the "zinc-containing layer."

The intensity distribution of $\mathrm{P}$ should have a different trend from that of $\mathrm{S}$ if the middle layer is missing in some part as observed by TEM in the previous study ${ }^{3)}$. From the result of this study, it is not clear if there is a difference because of the low intensity of $P$. The middle layer may exist only on the areas appear as orange in the optical image (Fig. 4) where the tribofilm is believed to be relatively thick. Although this point should be investigated further with other analytical methods, it may not be surprising if the middle layer had been mostly delaminated after repeated sliding. In fact, thin flake-like wear debris was observed in a different test with the same condition when the test was terminated after about tem minutes (not shown). The appearance of the wear debris was similar to that of the tribofilm on steel although no chemical analysis has been performed yet.

Dienwiebel and Pöhlmann ${ }^{7)}$ investigated the running-in process of metal pieces sliding in oil with ZDDP and reported that anti-wear ZDDP tribofilm covered on the sliding surface in a very early stage of friction and then disappeared from the surface after a short period. The onset of tribofilm delamination accompanied that of the reduction in friction coefficient. More interestingly, the fragments of the tribofilm were recognized underneath the surface. They suggest that such mechanical mixing modified the near surface volume, resulting in the friction reduction.

Similarly in this study, the middle layer would be partly delaminated to be the wear debris. The remaining part would be mechanically mixed with the initial surface (i.e. iron oxide) to form the zinc-containing layer that includes the elements of both the ZDDP and iron oxide. As reported in the previous study ${ }^{8)}$, the incorporation of zinc can be explained by the unique adsorption characteristics of ZDDP on the iron oxide. These give further explanation to the forming process of the layer by mechanical mixing that plays an important role to exhibit low friction.

By the way, it is reasonable to have a feature-less $\mathrm{O}$ distribution regardless of the existence of a phosphate layer similar to that on steel (i.e. the middle layer) because the initial surface of the plate specimen is covered by an iron oxide layer of few $\mu \mathrm{m}$ in thickness. The analytical depth of EPMA is $1 \mu \mathrm{m}$ or deeper, which is more than ten times deeper than the thickness of the tribofilm. Thus, strong signal of $\mathrm{O}$ from the iron oxide layer is to be detected everywhere resulting in feature-less image, so $\mathrm{O}$ was ignored in this study.

In future analyses, however, analyzing the lateral and spatial distributions of $\mathrm{O}$ with its chemical state by using more surface sensitive analytical technique such as XPS would bring more useful information. The $\mathrm{O}$ in the zinc-containing layer would be of phosphate and/or oxides. Generally speaking, ZDDP tribofilm on steel has phosphate glass structure and shows relatively high friction. On the other hand, specific type of oxide can exhibit relatively low friction. Similarly, sulfides exhibit low friction depending on the structure and composition. Therefore, similar analysis should be performed on $\mathrm{S}$ as well in order to discuss how the zinc-containing layer can be related to the low friction more specifically. 


\section{Conclusions}

Under the test condition of this study, the sliding pair of the iron oxide prepared by the water vapor treatment and the steel can achieve lower friction coefficient in PAO with 1 mass\% ZDDP than in pure PAO. The tribofilm on the iron oxide always includes the "zinc-containing layer" (i.e. a layer that includes $\mathrm{Zn}, \mathrm{Fe}$, $\mathrm{S}, \mathrm{P}$, and $\mathrm{O}$ ). Such tribofilm is distributed on the discolored area of wear track that covers almost entire contact surface.

It is suggested that the middle layer of the tribofilm is delaminated and partly mixed in the initial surface forming the zinc-containing layer that exhibits low friction.

\section{Acknowledgements}

A part of this study was supported by the 21 st century COE program, "International COE of Flow Dynamics," and NEDO Project No. 05A28003d. The water-vapor treatment for plates was made with the cooperation of Nippon Piston Ring, Co. Ltd. and Hino Motors, Ltd. ZDDP was supplied by Asahi Denka Co., Ltd. The tribotests were performed in Ecole Centrale de Lyon with cooperation by Prof. Jean-Michel Martin and Dr. Clotilde Minfray. The authors would like to express appreciation to those people and organizations.

\section{References}

[1] Takakura, T., Ishikawa, Y. and Ito, K., "The Wear Mechanism of Piston Rings and Cylinder Liners under Cooled-EGR Condition and the Development of Surface Treatment Technology for Effective Wear Reduction," SAE Paper 2005-01-1655, 2005, 1-12.

[2] Ishikawa, Y., Iwama, H., Naito, Y., Takakura, T., Ito, K. and Suzuki, T., "Combination of Cylinder Liner and Piston Ring of Internal Combustion Engine," U.S. Patent 6,553,957 B1, 2003.

[3] Ito, K., Minfray, C., Martin, J. M. and Kato, K., "Low Friction Tribofilm Formed by the Reaction of ZDDP on Iron Oxide," Tribology International, 39, 2006, 1538-1544.

[4] Dowson, D. and Higginson, G. R., "Lubrication and Wear: Fundamental and Application to Design," Proc. Inst. Mech. Eng., 182, Part 3A, 1968, 151-167.

[5] Dowson, D. and Higginson, G. R., "Elastrohydrodynamic Lubrication," Pergamon Press, NY, 1977.

[6] Timoshenko, S. and Goodier, J. N., "Theory of Elastisity," McGraw-Hill, NY, 1934.

[7] Dienwiebel, M. and Pöhlmann, K., "Nanoscale Evolution of Sliding Metal Surfaces during Running-in," Tribology Letters, 27, 2007, 255-260.

[8] Ito, K., Martin, J. M., Minfray, C. and Kato, K., "Formation Mechanism of a Low Friction ZDDP Tribofilm on Iron Oxide," Tribology Transactions, 50, 2007, 211-216. 